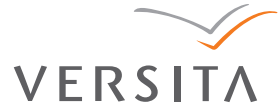

RESEARCH PAPER

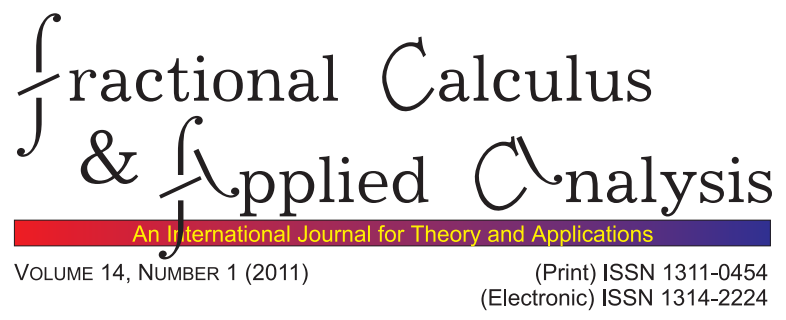

(Electronic) ISSN 1314-2224

\title{
FRACTIONAL DERIVATIVES IN SPACES \\ OF GENERALIZED FUNCTIONS
}

\author{
Mirjana Stojanović
}

\author{
Abstract \\ Dedicated to 80-th birthday of Prof. Rudolf Gorenflo
}

We generalize the two forms of the fractional derivatives (in RiemannLiouville and Caputo sense) to spaces of generalized functions using appropriate techniques such as the multiplication of absolutely continuous function by the Heaviside function, and the analytical continuation. As an application, we give the two forms of the fractional derivatives of discontinuous functions in spaces of distributions.

MSC 2010: 26A33, 46Fxx, 58C05

Key Words and Phrases: fractional derivatives, spaces of generalized functions, fractional derivatives of discontinuous function, analytical continuation

\section{Introduction}

Fractional Calculus (FC) is a field of mathematical analysis which deals integrals and derivatives of arbitrary order. FC is an old (cf. 44, 11], and references therein) as well as a new topic (cf. [10], 7], 20], [5], 8], 6], 9], [17] among the others). The main disadvantage of this calculus up to now, is the lack of appropriate functional spaces for their investigations. The first book in which the classical spaces of functions are considered is [16]. The recent book [7] is updated with some Real World's problems, where the fractional derivatives are involved in their modeling. This paper provides some contribution in that direction.

(c) 2011 Diogenes Co., Sofia

pp. 125-137, DOI: 10.2478/s13540-011-0009-5 
FC in spaces of distributions $\mathcal{S}_{+}^{\prime}$ and $\mathcal{D}_{+}^{\prime}$ as well as the distributions with compact support $\mathcal{E}^{\prime}$ is employed in papers [1], 2]. Two forms of fractional derivatives are embedded into the space of tempered distributions to obtain a framework for solving distributional diffusion-wave phenomena. FC is given in distributional setting and considered in a space of distributions over $\mathbb{R}$.

In this paper, we propose fractional derivatives of a discontinuous function to show how this distributional approach works. We extend the domain of orders $0<\alpha<1$, (resp. $m-1<\alpha<m, m \in \mathbb{N}$,) to the interval $\alpha<n+1$, (resp. $\alpha<n+m, n, m \in \mathbb{N}$ ), in which the fractional derivatives of absolutely continuous (resp. continuous) function are well-defined, using analytical continuation. The same is done for the regular distributions.

\section{Basic spaces}

\subsection{Space of absolutely continuous functions}

Let $L_{l o c}^{1}(\mathbb{R})$ be the space of locally integrable functions in $\mathbb{R} . A C^{m}([0, T])$, $T>0, m \in \mathbb{N}$, denotes the space of functions $f$, such that $f^{(k)}, k \in$ $\{0,1, \ldots, m-1\}$ are continuous and $f^{(m-1)}$ are absolutely continuous, i.e. $f^{(m)} \in L^{1}([0, T])$, (cf. [7]). In particular, $A C^{1}([0, T])=A C([0, T])$.

Denote by $C^{m}(\Omega)$ the space of functions $f$ which are $m$-times continuously differentiable on $\Omega$ supplied with the norm

$$
\|f\|_{C^{m}}=\sum_{k=0}^{m}\left\|f^{(k)}\right\|_{C}=\sum_{k=0}^{m} \max _{x \in \Omega}\left|f^{(k)}(x)\right|, \quad m \in \mathbb{N}_{0} .
$$

In particular, for $m=0, C^{0}(\Omega) \equiv C(\Omega)$ is the space of continuous functions $f$ on $\Omega$ with the norm $\|f\|_{C}=\max _{x \in \Omega}|f(x)|$.

\subsection{Space of distributions}

Denote by $P^{n} \subset \mathbb{R}^{n}$, whose elements have as coordinates entire nonnegative numbers. If $k=\left(k_{1}, \ldots, k_{n}\right) \in P^{n}$, then $|k|=k_{1}+\ldots+k_{m}$. We set $P^{1}=P, P=\mathbb{N}_{0}$.

Let $C_{0}^{m}(K), m \in P^{1}$, or $m=\infty$, where $K \subset \Omega$ is a compact, subset of $C_{0}^{m}(\Omega)$ whose elements have supports in $K$.

Define in the space $C_{0}^{\infty}(K)$, where $K$ is a compact subset of $\Omega$, the sequence of norms $\left\{p_{K, m} ; m \in P^{1}\right\}$ :

$$
p_{K, m}(\phi)=\sum_{|j| \leq m} \sup _{x \in K}\left|\phi^{(j)}(x)\right| .
$$

The vector space $C_{0}^{\infty}(K)$ supplied with this topology is a locally convex space $\mathcal{D}(K)$. The vector space $C_{0}^{\infty}(\Omega)$ supplied with the topology of strict inductive limit is a space $\mathcal{D}(\Omega)$. This space is the space of basic or test 
functions. A linear and continuous functional on $\mathcal{D}(\Omega)$ is a distribution. The distributions map $\mathcal{D}(\Omega)$ into $\mathbb{C}$ :

$$
f: \phi \mapsto<f, \phi>=(f, \bar{\phi})
$$

where $\bar{\phi}$ is the complex conjugate for the function $\phi$. For more details, cf. [13].

The regular distributions are distributions defined by locally integrable functions with the expression

$$
<f, \phi>:=\int_{\mathbb{R}^{n}} f(x) \phi(x) d x=\int_{K} f(x) \phi(x) d x, \text { where } K=\operatorname{supp} \phi, \text { from } \mathcal{D}^{\prime},
$$

where $f \in L_{l o c}^{1}(\Omega)$.

\subsection{Space of rapidly decreasing functions}

The space of rapidly decreasing functions which decrease faster than any polynomial, as $|x| \rightarrow \infty$, is denoted by $\mathcal{S}(\mathbb{R})$ (cf. [13]). The test space

$$
\mathcal{S}(\mathbb{R})=\left\{\phi \in C^{\infty}(\mathbb{R}) ; \quad \forall m, n \sup _{x \in \mathbb{R}}\left|x^{m} \phi^{(n)}(x)\right|<\infty\right\},
$$

is supplied with convergence given by the sequence of norms

$$
\|\phi\|_{k}=\sup _{\alpha \in\{0,1, \ldots, k\}}\left(1+|x|^{2}\right)^{k / 2}\left|\phi^{(\alpha)}(x)\right|, \quad k=0,1,2, \ldots
$$

A set of continuous linear functionals on $\mathcal{S}(\mathbb{R})$ with respect to the given sequence of norms $\|\cdot\|_{k}, k \in \mathbb{N}_{0}$, is the space of tempered distributions (space of slowly increasing functions) $\mathcal{S}^{\prime}(\mathbb{R})$. It holds $\mathcal{D}(\mathbb{R}) \subset \mathcal{S}(\mathbb{R})$, and consequently $\mathcal{S}^{\prime}(\mathbb{R}) \subset \mathcal{D}^{\prime}(\mathbb{R})$. When suppf $\subset \overline{\mathbb{R}}_{+}$, we deal with a space $\mathcal{S}_{+}^{\prime}\left(\overline{\mathbb{R}}_{+}\right)$. The test function space $\mathcal{S}_{0}(\mathbb{R})$ is a nonempty subspace of $\mathcal{S}(\mathbb{R})$ with the same topology induced by that in $\mathcal{S}(\mathbb{R})$. All functions in $\mathcal{S}(\mathbb{R})$ that vanish at the origin along with all of their derivatives are in $\mathcal{S}_{0}(\mathbb{R})$.

\section{From classical to distributional approach to the two forms of fractional derivatives}

For basic definitions and treatment of the Riemann-Louville and the Caputo derivative, cf. [16], [15], [7], 14], [12]. The Riemann-Liouville fractional integral of order $\alpha>0$ reads

$$
J^{\alpha} f(t):=\frac{1}{\Gamma(\alpha)} \int_{0}^{t}(t-\tau)^{\alpha-1} f(\tau) d \tau, \quad \alpha>0 .
$$

The Riemann-Liouville derivative of fractional order $D^{\alpha} f(t):=D^{m} J^{m-\alpha} f(t)$, where $m-1<\alpha \leq m, m \in \mathbb{N}$, is given by 
$D^{\alpha} f(t)=\left\{\begin{array}{l}\frac{d^{m}}{d t^{m}}\left[\frac{1}{\Gamma(m-\alpha)} \int_{0}^{t} \frac{f(\tau)}{(t-\tau)^{\alpha+1-m}} d \tau\right], \alpha \in(m-1, m), \\ \frac{d^{m}}{d t^{m}} f(t), \quad \alpha=m .\end{array} \quad m . \mathbb{N}\right.$.

The fractional derivative in the Caputo sense $D_{*}^{\alpha} f(t):=J^{m-\alpha} D^{m} f(t)$, where $m-1<\alpha \leq m, m \in \mathbb{N}$, is defined as follows:

$$
D_{*}^{\alpha} f(t)=\left\{\begin{array}{l}
\frac{1}{\Gamma(m-\alpha)} \int_{0}^{t} \frac{f^{(m)}(\tau)}{(t-\tau)^{\alpha+1-m}} d \tau, \quad \alpha \in(m-1, m), \quad m \in \mathbb{N} . \\
\frac{d^{m}}{d t^{m}} f(t), \quad \alpha=m .
\end{array}\right.
$$

The Riemann-Liouville and the Caputo fractional derivatives are well defined for an $f$ from the space $A C^{m}([0, T])$. But,

$$
D^{\alpha} f(t):=D^{m} J^{m-\alpha} f(t) \neq J^{m-\alpha} D^{m} f(t):=D_{*}^{\alpha} f(t),
$$

unless the function $f(t)$ along with its first $(m-1)$ derivatives vanish at $t=0^{+}$(cf. 44).

In order to extend the fractional derivatives to negative values of $t$, let us take sequence of functions which are smooth and defined (cf. [18)

$$
\eta_{\varepsilon}(t)= \begin{cases}1 & t>-a_{\varepsilon} \\ 0 & t<-2 a_{\varepsilon}\end{cases}
$$

$\eta_{\varepsilon} \in C^{\infty}(\mathbb{R}), \varepsilon>0$, then, $\forall \varepsilon \in(0, \infty), t \mapsto \eta_{\varepsilon}(t) f(t)$ has a support in $(-\infty, t), t<T$, for some $T>0, a_{\varepsilon}>0$ (cf. [13]). Define the generalized fractional derivative for $0<\alpha<1$, as

$$
D^{\alpha} f(t)=\frac{1}{\Gamma(1-\alpha)} \frac{d}{d t} \int_{-\infty}^{t} \frac{\eta_{\varepsilon}(\tau) f(\tau)}{(t-\tau)^{\alpha}} d \tau .
$$

This definition does not depend on the choice of $\varepsilon$ and $\eta_{\varepsilon}^{\prime}(t) \mapsto \delta(t)$, in $\mathcal{S}^{\prime}(\mathbb{R})$.

Recall the known results concerning the two forms of distributional fractional derivatives from [1, [2]. Since $f H$ is a regular distribution if an $f \in A C^{m}([0, T])$, where $H$ is the Heaviside function (cf. [19]), we can give an extension of the fractional derivatives to $t \in \mathbb{R}$. In distributional space $f \in \mathcal{S}_{+}^{\prime}$, or $\mathcal{D}_{+}^{\prime}$, the Riemann-Liouville and Caputo fractional derivatives equal,

$$
D^{\alpha} f(t):=D^{m} J^{m-\alpha} f(t) \equiv J^{m-\alpha} D^{m} f(t):=D_{*}^{\alpha} f(t) .
$$

We have the equality of the two forms of the distributional fractional derivatives of order $0<\alpha \in \mathbb{R}$ :

$$
D_{t}^{\alpha} f:=f_{-\alpha} * f\left(=f_{m-\alpha} * \frac{d^{m}}{d t^{m}} f=\frac{d^{m}}{d t^{m}}\left(f_{m-\alpha} * f\right)\right), \quad m \in \mathbb{N},
$$


for any $f \in \mathcal{S}_{+}^{\prime}(\mathbb{R})$, where the fractional operator $f_{m-\alpha}$ in the space of tempered distributions is defined as follows. For any $f_{\beta} \in \mathcal{S}_{+}^{\prime}, \beta \in \mathbb{R}$,

$$
f_{\beta}(x)=\left\{\begin{array}{cl}
\frac{H(t) t^{\beta-1}}{\Gamma(\beta)}, & \beta>0, \\
f_{\beta+N}^{(N)}(t), & \beta \leq 0, \quad \beta+N>0, \quad N \in \mathbb{N},
\end{array}\right.
$$

$(N)$ is the distributional derivative and $H$ is the Heaviside function. For evaluation of the fractional distributional derivatives, cf. also [18].

\section{Fractional derivatives of discontinuous function}

Recall from [3], p.31, the definition of the function continuous by parts which has the first derivative also continuous by parts, with discontinuity of the first kind at points $t_{1}, t_{2}, \ldots$ with jumps $h_{1}, h_{2}, \ldots$ The first derivative $f^{\prime}(t)$ is determined everywhere except at finite number of points. We shall find the derivative of the functional of the function $f$, which subordinates to the function $f(t)$. Define the function

$$
f_{1}(t)=f(t)-\sum_{k} h_{k} H\left(t-t_{k}\right), \quad f_{1}^{\prime}(t)=f^{\prime}(t)-\sum_{k} h_{k} \delta\left(t-t_{k}\right)
$$

and $f^{\prime}(t)=f_{1}^{\prime}(t)+\sum_{k} h_{k} \delta\left(t-t_{k}\right)$. If we suppose that the function $f(t)$ is continuous by parts with a continuous first derivative, then by differentiation, every discontinuous point $t_{k}$ of the function $f(t)$ with jumps $h_{k}$ adds to the expression of the derivative the term : $h_{k} \delta\left(t-t_{k}\right)$.

Let $\varphi \in C_{0}^{\infty}\left(\mathbb{R}_{+}\right), \varphi \geq 0, \int_{\mathbb{R}} \varphi(s) d s=1, \varphi(0)=0$, say a hat function. Suppose that we have only one discontinuous point $x_{k}$. The generalization for the finite number of them is then straightforward. Let $f(t)=f_{1}(t)+$ $h_{k} H\left(t-t_{k}\right)$. Then, $f^{\prime}(t)=f_{1}^{\prime}(t)+h_{k} \delta\left(t-t_{k}\right)$.

Proposition 4.1. Let $0<\alpha<1$, and $f(t)=f_{1}(t)+h_{k} H\left(t-t_{k}\right)$, where there exists only one discontinuous point $t_{k}>0$, with corresponding jump $h_{k}$. The distributional Riemann-Liouville fractional derivative of a discontinuous function does not correspond to the classical one, even in the case of the Caputo fractional derivative when $f(0)=0$. Compare (4) and (3i).

P r o o f. Find the classical Riemann-Liouville derivative of the discontinuous function $f$ with one discontinuity at a point $t_{k}$. We have

$$
\begin{aligned}
& D^{\alpha} f(t)=\frac{1}{\Gamma(1-\alpha)} \frac{d}{d t} \int_{0}^{t} \frac{f(\tau)}{(t-\tau)^{\alpha}} d \tau=\frac{1}{\Gamma(1-\alpha)}\left[\int_{0}^{t} \frac{f_{1}^{\prime}(\tau)+h_{k} \delta\left(\tau-t_{k}\right)}{(t-\tau)^{\alpha}}\right. \\
& \left.d \tau+\frac{f(0)+h_{k} H\left(-t_{k}\right)}{t^{\alpha}}\right]=\frac{1}{\Gamma(1-\alpha)}\left[\int_{0}^{t} \frac{f_{1}^{\prime}(\tau)}{(t-\tau)^{\alpha}} d \tau+\frac{f(0)}{t^{\alpha}}+h_{k} \frac{1}{\left(t-t_{k}\right)^{\alpha}}\right] .
\end{aligned}
$$


Thus, the ordinary Riemann-Liouville derivative of the discontinuous function with the discontinuity at the point $t_{k}$ is defined

$$
D^{\alpha} f(t)=\frac{1}{\Gamma(1-\alpha)}\left[\int_{0}^{t} \frac{f_{1}^{\prime}(\tau)}{(t-\tau)^{\alpha}} d \tau+\frac{f(0)}{t^{\alpha}}+\frac{h_{k}}{\left(t-t_{k}\right)^{\alpha}}\right] .
$$

This definition does not correspond to the definition in distributional sense (cf. (4)).

Consider the distributional Riemann-Liouville fractional derivative for $0<\alpha<1$, of discontinuous function with the discontinuity at point $t_{k}$. We have

$$
\begin{gathered}
<D^{\alpha} f(t), \varphi(t)>=-<f(t), D^{\alpha} \varphi(t)>=-<f_{1}(t)+h_{k} H\left(t-t_{k}\right), \frac{1}{\Gamma(1-\alpha)} \\
\frac{d}{d t} \int_{0}^{t} \frac{\varphi(\tau)}{(t-\tau)^{\alpha}} d \tau>=<f_{1}^{\prime}(t), \int_{0}^{t} \frac{\varphi(\tau)}{(t-\tau)^{\alpha}} d \tau>+\frac{1}{\Gamma(1-\alpha)} h_{k}<\delta\left(t-t_{k}\right) \\
\int_{0}^{t} \frac{\varphi(\tau)}{(t-\tau)^{\alpha}} d \tau>\leq<\int_{0}^{t} \frac{f_{1}^{(1)}(\tau)}{(t-\tau)^{\alpha}} d \tau, \varphi(\tau)>+C .
\end{gathered}
$$

Thus, for discontinuous function $f$ with discontinuity point $t_{k}$ we have the following expression for the Riemann-Liouville distributional fractional derivative

$$
D^{\alpha} f=\frac{1}{\Gamma(1-\alpha)}\left[\int_{0}^{t} \frac{f_{1}^{(1)}(\tau)}{(t-\tau)^{\alpha}} d \tau\right]
$$

Similar situation arises when the function $f$ has finite number of discontinues points $t_{1}, t_{2}, \ldots$ where all discontinuities are positive or equal zero. Then, we have

$$
f(t)=f_{1}(t)-\sum_{k} h_{k} H\left(t-t_{k}\right), \quad f^{\prime}(t)=f_{1}^{\prime}(t)-\sum_{k} h_{k} \delta\left(t-t_{k}\right) .
$$

Repeating the same procedure for the distributional Riemann-Liouville fractional derivative for $0<\alpha<1$, with one discontinuity, we obtain

$$
D^{\alpha} f(t)=\frac{1}{\Gamma(1-\alpha)}\left[\int_{0}^{t} \frac{f_{1}^{(1)}(\tau)}{(t-\tau)^{\alpha}} d \tau\right] .
$$

Recall that for the ordinary Riemann-Liouville derivative of the discontinues function at finite number of discontinues points in classical spaces (say $f \in A C^{1}([0, T])$, we have

$$
D^{\alpha} f(t)=\frac{1}{\Gamma(1-\alpha)}\left[\int_{0}^{t} \frac{f_{1}^{(1)}(\tau)}{(t-\tau)^{\alpha}} d \tau+\frac{f(0)}{t^{\alpha}}+\sum_{k} \frac{h_{k}}{\left(t-t_{k}\right)^{\alpha}}\right] .
$$


We have as in (3) for the discontinuity point $t_{k}, t_{k}>0$, and consequently for finite sum of discontinuities $t_{1}, t_{2}, \ldots$, with negative jumps extended to negative values of $t$

$$
D^{\alpha} f(t)=\frac{1}{\Gamma(1-\alpha)}\left[\int_{-\infty}^{t} \frac{f_{1}^{(1)}(t) \eta_{\varepsilon}(\tau)}{(t-\tau)^{\alpha}} d \tau-\sum_{k} h_{k} \frac{\eta_{\varepsilon}\left(t_{k}\right)}{\left(t-t_{k}\right)^{\alpha}}+\frac{f_{1}(0)}{t^{\alpha}}\right] .
$$

Consider a regularization for the function $f$ which have a singularity at the point $0 \in \Omega \subset \mathbb{R}$. Recall from [13]:

THEOREM 4.1. ([13]) If $\tilde{f}(x)$ is a regularization for the function $f$ which have a singularity at the point $0 \in \Omega \subset \mathbb{R}$, then $\tilde{f}(x)+\sum_{|k| \leq r} c_{k} \delta^{(k)}$ for arbitrary $r \in \mathbb{N}$ and arbitrary complex constants $c_{k},|k| \leq r$, is also regularization of $f$.

Considering the fractional derivative of a regularization $\tilde{f}$ (resp. $\tilde{f}+$ $\left.\sum_{|k| \leq r} c_{k} \delta^{(k)}\right)$ we conclude the following.

Proposition 4.2. The Riemann-Liouville and the Caputo fractional derivatives for a regularization $\tilde{f}$ at zero, (resp. for $\tilde{f}(x)+\sum_{|k| \leq r} c_{k} \delta^{(k)}$ ) from Theorem 4.1 equal in distributional sense, resp. all singularities at point zero vanish for appropriately chosen test function $\varphi \in \mathcal{D}_{+}$, (resp. $\left.\varphi \in \mathcal{S}_{0}\right)$.

$$
\begin{aligned}
& \text { P r o o f. We have for } 0<\alpha<1, \\
& \left(D^{\alpha} \tilde{f}, \varphi>=-\frac{1}{\Gamma(1-\alpha)}\left[<\tilde{f}+\sum_{|k| \leq r} c_{k} \delta^{(k)}(t), \frac{d}{d t} \int_{0}^{t} \frac{\varphi(\tau)}{(t-\tau)^{\alpha}} d \tau>\right]\right. \\
& =-\frac{1}{\Gamma(1-\alpha)}\left[<\int_{0}^{t} \frac{\tilde{f}^{(1)}}{(t-\tau)^{\alpha}} d \tau, \varphi>+\sum_{|k| \leq r} c_{k}(-1)^{k+1} \frac{\sup _{t \in[0, T]}\left|\varphi^{(k+1)}(t)\right|}{(1-\alpha) t^{\alpha-1}}\right. \\
& \left.+\frac{\varphi(0)}{t^{\alpha}}+\frac{\varphi^{(1)}(0)}{t^{\alpha}}+\ldots+\frac{\varphi^{(k)}(0)}{t^{\alpha}}\right]=-\frac{1}{\Gamma(1-\alpha)}\left[<\int_{0}^{t} \frac{\tilde{f}^{(1)}}{(t-\tau)^{\alpha}} d \tau, \varphi>\right] .
\end{aligned}
$$

Since the function $\tilde{f}$ is a regularization and $\varphi \in C_{0}^{\infty}\left(\mathbb{R}_{+}\right), \varphi \geq 0, \int_{\mathbb{R}} \varphi(x) d x$ $=1$, it is allowed to apply the Fubini-Tonelli theorem.

If we choose such test function $\varphi$, that $\varphi(0)=\varphi^{(1)}(0)=\ldots=\varphi^{(k+1)}(0)$ $=0$, where $|k| \leq r$, (say hat function massed at a point $t_{0}>0$ ), we obtain the assertion. Another possibility is to take $\varphi \in \mathcal{S}_{0}$. 
We shall find fractional derivative extended to negative values of discontinuous function for the piecewise discontinuous function $f(t)=f_{1}(t)-$ $h_{k} H\left(t-t_{k}\right), t_{k}>0$.

Proposition 4.3. Let a piecewise discontinuous function $f(t)=f_{1}(t)$ $-h_{k} H\left(t-t_{k}\right)$, where $f \in A C^{1}([0, T])$, (resp. $\left.f \in A C^{m}([0, T]), m \in \mathbb{N}\right)$ have a discontinuity at a point $t_{k}, t_{k}>0$ (resp. finite number of discontinuities).

(a) In the classical sense, we consider a discontinuity point as a singularity. The Riemann-Liouville fractional derivative $D^{\alpha} f$, with discontinuous point $t_{k}>0$, and jump $h_{k}$, has the form (3) for one discontinuity (resp. the form (5) for finite number of discontinuities).

(b) In distributional sense, in the space $\mathcal{D}_{+}^{\prime}$, (resp. $\left.\mathcal{S}_{+}^{\prime}\right)$, the discontinuity points in the Riemann-Liouville and the Caputo fractional derivatives have not influence to the form of derivatives, i.e. fractional derivative at a point of discontinuity is neutral element with respect to the operation of the fractional differentiation in the spaces of distributions $\mathcal{S}_{+}^{\prime}$, (resp. $\left.\mathcal{D}_{+}^{\prime}\right)$.

P r o o f. Suppose that $f \in A C^{1}([0, T])$, (resp. $f \in A C^{m}([0, T]), m \in$ $\mathbb{N})$. For the first assertion, cf. Proposition 4.1. For the second assertion in the space of distributions $\mathcal{S}^{\prime}$ (resp. $\mathcal{D}^{\prime}$ ), we have in distributional sense that the Riemann-Louvile fractional derivative equals the Caputo fractional derivative and they read for $0<\alpha<1$, and finite number of discontinuous points

$$
D_{*}^{\alpha} f(t)=D^{\alpha} f(t)=\frac{1}{\Gamma(1-\alpha)}\left[\int_{-\infty}^{t} \frac{f_{1}^{(1)}(t) \eta_{\varepsilon}(\tau)}{(t-\tau)^{\alpha}} d \tau\right],
$$

where $\eta_{\varepsilon}$ is defined in Section 3. In general, for $m-1<\alpha<m, m \in \mathbb{N}$,

$$
D_{a^{+}}^{\alpha} f(t)=D_{* a^{+}}^{\alpha} f=\frac{1}{\Gamma(m-\alpha)}\left[\int_{-\infty}^{\infty} \frac{\left(f^{(m)}(\tau) H(\tau-a)\right)}{(t-\tau)^{\alpha+1-m}}\right], a \in \mathbb{R},
$$

in distributional sense.

Invariant properties of the fractional differentiation in distributional spaces with respect to discontinuous points are the consequence of the property of the invariancy of distributional spaces with respect to the operation generated by a polynomial.

\section{Regularization via analytical continuation}

In the sequel, we shall use regularization via analytical continuation from [13], 3]. Let $0<\alpha<1$. With analytical continuation, we have for 
the Riemann-Liouville fractional derivative of an $f(t) \in A C^{1}([0, T])$

$$
\begin{gathered}
D^{\alpha} f(t)=\frac{1}{\Gamma(1-\alpha)}\left[\int_{0}^{1} \frac{\left(f^{(1)}(\tau)-f^{(1)}(0)\right)}{(t-\tau)^{\alpha}} d \tau+\int_{1}^{t} \frac{f^{(1)}(\tau)}{(t-\tau)^{\alpha}} d \tau\right. \\
\left.+\frac{f(0)}{t^{\alpha}}+\frac{f^{(1)}(0)}{1-\alpha}\right] .
\end{gathered}
$$

The first integral is defined for $\alpha<2$, the second for every $\alpha$, and the last expression is defined for $\alpha \neq 1$. The only discontinuity is due to the term $\frac{f(0)}{t^{\alpha}}$ for $t=0$. For the Caputo derivative (since $f(0)=0$ ), there is no discontinuities and fractional derivative is regular for $\alpha<2$, $\alpha \neq 1$. We extend the analytical distribution $(t-\tau)_{+}^{-\alpha}, \alpha<1$, by analytical continuation to the area $\alpha<2, \alpha \neq 1$, (cf. [13]).

Proposition 5.1. Let $f \in \mathcal{S}_{+}^{\prime}$ (resp. $\mathcal{D}_{+}^{\prime}$ ) be a regular distribution. Then, the analytical continuation extends the region for the derivative orders from $0<\alpha<1$, to $\alpha<2, \alpha \neq 1$, because of the distributional representation

$$
D^{\alpha} f=\frac{1}{\Gamma(1-\alpha)}\left[\int_{0}^{1} \frac{\left(f^{(1)}(\tau)-f^{(1)}(0)\right)}{(t-\tau)^{\alpha}} d \tau+\int_{1}^{t} \frac{f^{(1)}(\tau)}{(t-\tau)^{\alpha}} d \tau\right] .
$$

P r o o f. Since $f H$ is a regular distribution when $f \in A C^{1}([0, T])$, $0<\alpha<1$, we have for an $f \in A C^{1}([0, T]), 0<\alpha<1$,

$$
\begin{gathered}
D_{+0}^{\alpha} f(t)=\frac{1}{\Gamma(1-\alpha)}\left[\int_{0}^{1} \frac{\left(f^{(1)}(\tau)-f^{(1)}(0)\right) H(\tau)}{(t-\tau)^{\alpha}} d \tau+\int_{1}^{t} \frac{f^{(1)}(\tau) H(\tau)}{(t-\tau)^{\alpha}} d \tau\right. \\
\left.+\frac{f(0) H(0)}{t^{\alpha}}+\frac{f^{(1)}(0)}{1-\alpha}\right] .
\end{gathered}
$$

In distributional sense,

$$
D_{+0}^{\alpha} f(t)=\frac{1}{\Gamma(1-\alpha)}\left[\int_{0}^{1} \frac{\left(f^{(1)}(\tau)-f^{(1)}(0)\right) H(\tau)}{(t-\tau)^{\alpha}} d \tau+\int_{1}^{t} \frac{f^{(1)}(\tau) H(\tau)}{(t-\tau)^{\alpha}} d \tau\right]
$$

and the assertion follows since $f H$ is a regular distribution for $f \in A C^{1}([0, T])$.

Continuation of this technique (cf. [13, [3]), for the Riemann-Liouville derivative for $0<\alpha<1$, reads

$$
\begin{gathered}
D^{\alpha} f(t)=\frac{1}{\Gamma(1-\alpha)}\left[\int_{0}^{1} \frac{\left(f^{(1)}(\tau)-f^{(1)}(0)-\ldots-\frac{\tau^{n-1}}{(n-1) !} f^{(n)}(0)\right)}{(t-\tau)^{\alpha}} d \tau\right. \\
\left.+\int_{1}^{t} \frac{f^{(1)}(\tau)}{(t-\tau)^{\alpha}} d \tau+\sum_{k=1}^{n} \frac{f^{(n)}(0)}{(k-1) !(-\alpha+k)}+\frac{f(0)}{t^{\alpha}}+R_{n}\right],
\end{gathered}
$$


where $R_{n}$ is a regular part if $\alpha \notin \mathbb{N}$. The first integral is defined for $\alpha<n+1$, the second for every $\alpha$ and the third expression for $\alpha \notin \mathbb{N}$. By this regularization we extend the domain from $0<\alpha<1$ to $\alpha<n+1, \alpha \notin \mathbb{N}$. Now, by analytical continuation the Riemann-Liouville-fractional derivative defined almost everywhere for $f \in A C^{n}([0, T]), T>0$, is extended to the orders $\alpha<n+1, n \in \mathbb{N}, \alpha \notin \mathbb{N}$. In particular, by analytical continuation, the Riemann-Liouville derivative is defined on an interval $[0, T]$ almost everywhere for $\alpha<2$, if $f \in A C([0, T]), T>0$, (resp. $f$ is a regular distribution). We exclude the entire points (here point 1 ).

Following the same lines as in Proposition [5.1, we arrive at

Proposition 5.2. Suppose that $f \in \mathcal{S}_{+}^{\prime}$ (resp. $\mathcal{D}_{+}^{\prime}$ ) be a regular distribution, and $0<\alpha<1$. Then, the Riemann-Liouville fractional derivative $D^{\alpha} f$ is well defined almost everywhere in $[0, T], T>0$, for $\alpha<1+n$, $n \in \mathbb{N}$, except in entire points $\alpha \in \mathbb{N}$.

P r o o f. The written formula reads for $f \in A C^{n}([0, T])$. The analytical continuation of the Riemann-Liouville fractional derivative in spaces of distributions reads

$$
\begin{gathered}
D^{\alpha} f(t)=\frac{1}{\Gamma(1-\alpha)}\left[\int_{0}^{1} \frac{\left(f^{(1)}(\tau)-f^{(1)}(0)-\ldots-\frac{\tau^{n-1}}{(n-1) !} f^{(n)}(0)\right)}{(t-\tau)^{\alpha}} d \tau\right. \\
\left.+\int_{1}^{t} \frac{f^{(1)}(\tau)}{(t-\tau)^{\alpha}} d \tau\right],
\end{gathered}
$$

what gives the sufficient and necessary condition for the existence of the Riemann-Liouville fractional derivative almost everywhere in interval $[0, T]$, for $T>0$. In distributional sense the extension is given to the area $\alpha<$ $1+n, n \in \mathbb{N}, \alpha \notin \mathbb{N}$. Only the integrals in the expression for $D^{\alpha} f$ remain.

Suppose that $f \in A C^{m+n}([0, T]), T>0$. Then, the Riemann-Liouville fractional derivative given with (11) is defined almost everywhere in $[0, T]$, $T>0$, for $m-1 \leq \alpha<m, m \in \mathbb{N}$, (cf. [7]). The analytical continuation reads

$$
\begin{aligned}
& D^{\alpha} f(t)=\frac{d^{m}}{d t^{m}}\left[\frac{1}{\Gamma(m-\alpha)} \int_{0}^{t} \frac{f(\tau) d \tau}{(t-\tau)^{\alpha+1-m}}\right] \\
& \quad=\frac{1}{\Gamma(m-\alpha)}\left[\int_{0}^{t} \frac{f^{(m)}(t-\tau) d \tau}{\tau^{\alpha+1-m}}+\sum_{k=0}^{m-1} \frac{f^{(k)}(0)}{t^{\alpha+1-k}}\right],
\end{aligned}
$$

where we denote

$$
I_{1}=\frac{d^{m}}{d t^{m}} \frac{1}{\Gamma(m-\alpha)}\left[\int_{0}^{1} \frac{\left(f(\tau)-f(0)-\ldots-\frac{\tau^{(n-1)}}{(n-1) !} f^{(n)}(0)\right)}{(t-\tau)^{\alpha+1-m}} d \tau\right.
$$




$$
\begin{gathered}
\left.+\int_{1}^{\infty} \frac{f(\tau)}{(t-\tau)^{\alpha+1-m}} d \tau+\sum_{k=1}^{m} \frac{f^{(k-1)}(0)}{(k-1) !(-\alpha+k)}\right] \\
I_{2}=\frac{1}{\Gamma(m-\alpha)}\left[\int_{0}^{1} \frac{\left(f^{(m)}(\tau)-f^{(m)}(0)-\ldots-\frac{\tau^{(n-1)}}{(n-1) !} f^{(m+n)}(0)\right)}{(t-\tau)^{\alpha+1-m}} d \tau\right. \\
\left.+\int_{1}^{\infty} \frac{f^{(m)}(\tau)}{(t-\tau)^{\alpha+1-m}} d \tau+\sum_{k=1}^{n} \frac{f^{(m+k-1)}(0)}{(k-1) !(-\alpha+k+m)}+\sum_{k=0}^{m-1} \frac{f^{(k)}(0)}{t^{\alpha+1-k}}\right] .
\end{gathered}
$$

In $I_{1}$ (resp. $I_{2}$ ) the first integral is defined for $\alpha<n+1$, (resp. for $\alpha<n+m)$, the second for every $\alpha$ and the third for $\alpha \notin \mathbb{N}$. By this regularization we extend the domain of $I_{1}$ (resp. $I_{2}$ ) from $0<\alpha<1$ to $\alpha<n+1$, (resp. from $m-1<\alpha<m$, to $\alpha<n+m$ ), $\alpha \notin \mathbb{N}$.

Thus, by analytical continuation the Riemann-Liouville fractional derivative defined almost everywhere for the function $f \in A C^{m+n}([0, T])$, $T>0$, for the orders $m-1<\alpha<m, m \in \mathbb{N}$, is extended to the orders $\alpha<n+m, \alpha \notin \mathbb{N}, n, m \in \mathbb{N}$. The discontinuity is at the point zero. For $f(0)=f^{(1)}(0)=\ldots=f^{(m-1)}(0)=0, m \in \mathbb{N}$, we obtain the Caputo derivative, i.e. the cut-off regularization of the Riemann-Liouville derivative at a point zero which leads to the regularization at zero.

In distributional sense in these formulas for $D^{\alpha} f$ we keep only the integral summands. The extension area is $\alpha<n+m, \alpha \notin \mathbb{N}, n, m \in \mathbb{N}$.

Concerning the distributional approach, we have the following

Proposition 5.3. Let $f \in \mathcal{S}_{+}^{\prime}$, or $f \in \mathcal{D}_{+}^{\prime}$, be a regular distribution. Let $m-1<\alpha<m, m \in \mathbb{N}$. Then, Riemann-Liouville fractional derivative $D^{\alpha} f$ is defined almost everywhere in interval $[0, T], T>0$, for derivative orders $\alpha<m+n, m, n \in \mathbb{N}$, when $\alpha \notin \mathbb{N}$, more precisely when $\alpha \neq$ $1,2, \ldots, k+m, k=1, \ldots, n, m \in \mathbb{N}$.

P r o o f. Analytical continuation in the space of distributions, (cf. $I_{2}$ ), yields

$$
\begin{gathered}
D^{\alpha} f(t)=\frac{1}{\Gamma(m-\alpha)}\left[\int_{0}^{1} \frac{\left(f^{(m)}(\tau)-f^{(m)}(0)-\ldots-\frac{\tau^{(n-1)}}{(n-1) !} f^{(m+n)}(0)\right)}{(t-\tau)^{\alpha+1-m}} d \tau\right. \\
\left.\quad+\int_{1}^{\infty} \frac{f^{(m)}(\tau)}{(t-\tau)^{\alpha+1-m}} d \tau\right], \quad m, n \in \mathbb{N}, m-1<\alpha<m, \alpha \neq \mathbb{N},
\end{gathered}
$$

what gives the range of orders $\alpha, \alpha<m+n, \alpha \neq \mathbb{N}$.

Repeating the procedure given for the Riemann-Liouville-derivative, we conclude the next for the Caputo fractional derivative.

Proposition 5.4. Suppose $\alpha>0, m-1<\alpha<m, m \in \mathbb{N}$. If $f \in A C^{m+n}([0, T]), T>0$, then the Caputo fractional derivative exists 
almost everywhere in $[0, T], T>0$, when $m-1<\alpha<m, m \in \mathbb{N}$. If $\alpha \notin \mathbb{N}_{0}$, by analytical continuation the orders of $\alpha$ can be extended to the interval $\alpha<n+m, n, m \in \mathbb{N}$. In this way, the Caputo fractional derivative exists almost everywhere in $[0, T], T>0$, if $f \in A C^{m+n}([0, T]), T>0$, for $\alpha<n+m, n, m \in \mathbb{N}$. In particular, for $0<\alpha<1$, the extension of the orders is given to the interval $\alpha<n+1, n \in \mathbb{N}$.

Applying Theorem 2.2 from [7], we have the next proposition.

Proposition 5.5. Let $\alpha \geq 0$, and $m-1<\alpha<m, m \in \mathbb{N}$. Let $f \in C^{m+n}([0, T]), T>0$. Then, the Caputo fractional derivative with orders $m-1<\alpha<m, m \in \mathbb{N}$, is continuous in [0,T], T>0. If $\alpha \notin \mathbb{N}_{0}$, the Caputo fractional derivative is continuous for $\alpha<m+n, m, n \in \mathbb{N}$, via analytical continuation.

Proposition 5.6. Suppose $m-1<\alpha<m, m \in \mathbb{N}$. Using analytical continuation, the Caputo fractional derivative is bounded from the space $C^{n+m}([0, T])$, to the space $C^{0}([0, T])$ and the following estimate holds

$$
\left\|D^{\alpha} f\right\|_{C^{0}} \leq \frac{T^{n+m-\alpha}}{\Gamma(m-\alpha)(n+m-\alpha+1)},
$$

i.e. this property given for $m-1<\alpha<m, m \in \mathbb{N}$, is extended to the orders $\alpha<n+m, n, m \in \mathbb{N}$.

The other properties of the Riemann-Liouville (resp. Caputo) fractional derivative can be extended similarly to the higher orders of $\alpha$ using analytical continuation.

\section{References}

[1] T. Atanacković, S. Pilipović, D. Zorica, Time distributed order diffusion-wave equation, I: Volterra type equation. Proc. R. Soc. A 465 (2009), 1869-1891.

[2] T. Atanacković, S. Pilipović, D. Zorica, Time distributed order diffusion-wave equation, $I I$ : Application of Laplace and Fourier transforms. Proc. R. Soc. A 465 (2009), 1893-1917.

[3] I.M. Gelfand, G.E. Shilov, Generalized Functions and Operations, Vol. 1. Physics-Math. Lit., Moscow, 1959 (In Russian).

[4] R. Gorenflo, F. Mainardi, Fractional calculus: Integral and differential equations of fractional order. Chapter in: A. Carpinteri and F. Mainardi(Eds.): Fractals and Fractional Calculus in Continuum Mechanics, Springer-Verlag, Wien and New York, 1997, 223-276.

[5] R. Gorenflo, Yu.F. Luchko, and S.R. Umarov, On the Cauchy and multi-point problems for partial pseudo-differential equations of fractional order. Fract. Calc. Appl. Anal. 3, No 3 (2000), 249-275. 
[6] G. Jumarie, An approach via fractional analysis to non-linearity induced by coarse-graining in space. Nonlinear Analysis: Real World Applications 11, No 1 (2010), 535-546.

[7] A. Kilbas, H.M. Srivastava, J.J. Trujillo, Theory and Applications of Fractional Differential Equations. North-Holland, Elsevier, 2006.

[8] V. Lakshmikantham, S. Leela, Devi Vasundhara, Theory of Fractional Dynamic Systems. Cambridge Academic Publishers, Cambridge, 2009.

[9] C.F. Lorenzo, T.T. Hartley, Initialization of fractional differential equations: Theory and applications. In: Proc. of the ASME' 2007 International Design Engineering Technical Conference, DETC2007-34814, Las Vegas, USA, 2007.

[10] F. Mainardi, Fractional Calculus and Waves in Linear Viscoelasticity. Imperial College Press \& World Scientific Co. Inc., 2010.

[11] F. Mainardi, Fractional calculus: Some basic problems in continuum and statistical mechanics. In: A. Carpinteri, F. Mainardi (Eds.), CISM Courses and Lecture Notes, No 378, 1997, 291-348.

[12] F. Mainardi, A. Mura, R. Gorenflo, M. Stojanović, The two forms of fractional relaxation of distributed order. J. Vibration and Control 13 (2007), 1249-1268.

[13] S. Pilipović, B. Stanković, The Spaces of Distributions. Serbian Academy of Science, Novi Sad, 2000.

[14] I. Podlubny, Fractional Differential Equations. Mathematics in Science and Engineering 198, Academic Press, 1999.

[15] B. Rubin, Fractional Integrals and Potentials. Addison Wesley Longman Ltd., Harlow, 1996.

[16] S.G. Samko, A.A. Kilbas, O.I. Marichev, Fractional Integrals and Derivatives. Gordon and Beach, Amsterdam, 1993.

[17] M. Stojanović, Foundations of fractional calculus in generalized functions algebras. Analysis and Applications, To appear (2011).

[18] M. Stojanović, Note on fractional distributional derivatives. Preprint (2010).

[19] V.S. Vladimirov, Generalized Functions in Mathematical Physics. Mir Publishers, Moscow, 1979.

[20] S. Umarov, R. Gorenflo, Cauchy and nonlocal multi-point problems for distributed order pseudo-differential equations, Part One. J. Anal. Appl. 245, No 3 (2005), 449-466.

Department of Mathematics and Informatics, University of Novi Sad Trg D.Obradovića 4, 21000 Novi Sad, SERBIA 